\title{
Modeling and Analysis of Regional Registration Based Mobile Multicast Service Management
}

\author{
Ing-Ray Chen \\ Department of Computer Science \\ Virginia Tech \\ irchen@cs.vt.edu
}

\author{
Ding-Chau Wang \\ Department of Information Management \\ Southern Taiwan University of Technology \\ zh9@mail.stut.edu.tw
}

\begin{abstract}
We propose and analyze a regional registration based mobile multicast service management scheme which we call URRMoM for efficiently supporting mobile multicast in Mobile IP networks. URRMoM extends the design concept of Remote Subscription (RS) by using mobile multicast agents (MMAs), each covering a number of MHs in its service area through tunneling multicast packets received from the multicast source to them. URRMoM adopts a user-central design allowing each mobile host to determine its optimal MMA service area dynamically depending on its service and mobility characteristics. We develop an analytical model to describe the behavior of a MH operating under URRMoM and derive the optimal service area of the MMA based on the MH's service and mobility characteristics to minimize the total network traffic generated due to multicast packet delivery and multicast tree maintenance. We present analytical results validated by simulation to demonstrate the benefit of our proposed regional registration based mobile multicast service management scheme over existing schemes including RS and RBMoM.
\end{abstract}

Index Terms - Mobile IP, multicast, mobility handoff, performance analysis, regional registration.

\section{INTRODUCTION}

$\mathrm{M}^{-}$ ULTICASTING for delivering data from a source node to multiple nodes is widely used to support applications such as broadcasting services, voice or video conferencing and database replication. An ideal mechanism for multicast should meet the requirements of delivering a copy of the same massage to multiple destination nodes while keeping duplicate data and the distance traveled to the minimum. In mobile IP environments, providing multicast support to mobile hosts (MHs) is challenging because a mobile multicast protocol must consider not only dynamic group membership but also dynamic member topology.

Two basic schemes have been proposed for Mobile IP networks [3] to support mobile multicast. In the remote subscription (RS) scheme, a mobile host (MH) always needs to subscribe to its multicast group when it enters or changes a foreign network. That is, whenever the $\mathrm{MH}$ moves from one network to another, its local router must be added to the multicast tree. The update frequency to the multicast tree thus is proportional to the handoff frequency of $\mathrm{MH}$. The advantage of this approach is that the data in the multicast tree are always delivered on optimal shortest paths. The disadvantage is the extra overhead for reconstructing the multicast tree whenever a mobility handoff occurs. This scheme does not handle the situation of source mobility.

In the bi-directional tunneling (BT) scheme, a $\mathrm{MH}$ receives multicast data by way of its home network using unicast mobile IP tunnels from its Home Agent (HA). The advantage of this approach is that it handles not only recipient mobility but also source mobility. There is no need to update the multicast delivery tree when a MH's location is changed. The main disadvantage of this scheme is that the routing path for multicast packet delivery may be far from optimal. Another drawback is that it must replicate and deliver tunneled multicast packets to each of the $\mathrm{MH}$ group members regardless of which foreign networks they reside in. Thus, it offers limited scalability.

Various approaches have been proposed to combine the advantages of RS and BT for mobile multicast in IP networks. Our approach also attempts to combine the advantages of RS and BT. It extends the design concept of per-user regional registration [1] for integrated mobility and service management for unicast services to mobile multicast services. The objective is to demonstrate that each $\mathrm{MH}$ can autonomously determine its optimal service area under regional registration based on its service and mobility characteristics such that the overall network traffic generated due to multicast tree maintenance (because of user mobility) and multicast packet delivery (because of multicast service) can be minimized.

The rest of the paper is organized as follows. In Section II we survey existing work and comment on the relation to our work. In Section III we describe our proposed $\underline{\text { User-oriented }}$

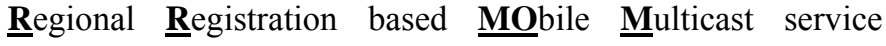
management scheme abbreviated as URRMoM for providing efficient mobile multicast services in mobile IP environments. In Section IV, we develop a performance model to evaluate the performance characteristics of our proposed scheme. Section $\mathrm{V}$ presents numerical results obtained from evaluating the performance model. We show that there exists an optimal 
mobile multicast agent service area under which the overall traffic generated for multicast packet delivery and multicast tree maintenance will be minimized. We demonstrate that our approach outperforms the basic RS scheme and also an existing hybrid scheme RBMoM [2]. In Section VI we report simulation results compared against analytical results for simulation validation. Finally in Section V we conclude the paper and outline some future research areas.

\section{RELATED WORK}

Local registration to support multicast services was proposed to allow MNs to send registration requests to a regional agent [4]. The approach is similar to regional registration for mobility management [1] except that multicast services are being considered. The concept of multicast agent (MA) was investigated [6] to provide multicast services to mobile group members in multiple foreign networks. Each MA manages a list of multicast groups that have mobile members in its service area. An MA receives multicast packets for a group, and then tunnels them to foreign agents (FAs) that host visiting MHs in the group. In this approach, MAs serve as access points to the multicast backbone and are placed near to FAs to reduce the tunneling distance. This scheme has a relatively stable structure and avoids frequent modifications to the multicast tree as the tree points to MAs. However, it lacks flexibility to adapt to individual MH's mobility patterns since MAs are static, which causes large network traffic in multicast packet delivery and suffers a single point of failure problem.

Recently, a hybrid approach of BT and RS, called mMOM, was proposed in [5]. A MH in mMOM applies either BT or RS based on its mobility. If the MH is highly mobile, BT will be used. Otherwise RS will be used. Every MH must reregister with its foreign agent (FA) after a period of residence time. The FA then decides if a $\mathrm{MH}$ is mobile or immobile based on if it receives the re-register message. mMOM approach is simple and practical. However, the approach does not allow co-located care of address to be used as in MIP.

RBMoM [2] employs a mobile multicast agent (MMA) to tunnel multicast packets to the FA to which the MH is currently attached. The information about which MMA is currently serving a $\mathrm{MH}$ is recorded at the MH's HA. If a MH is out of the current MMA's service range, then a MMA handoff occurs and another MMA will take over the multicast service for the MH. One problem with RBMoM is that when a FA subscribes to the multicast tree serving as a MMA, it needs to send an update message to all FAs in the MMA range to inform them of this subscription information, so these FAs can each update its agent table to know what MMAs are around. Then, each time a MH moves into the area of a FA, it can check the FA's agent table to choose the nearest MMA as its MMA. This incurs unnecessary communication overheads and performance penalty to the network.

Our paper proposes a user-oriented regional registration based mobile multicast protocol (URRMoM) to allow each $\mathrm{MH}$ to autonomously determine its new MMA when it moves out of the current MMA's regional area. This is achieved without the overhead of maintaining the agent table kept at each FA as in URRMoM. In addition, the optimal regional registration area size is determined dynamically by each $\mathrm{MH}$ based on its dynamic mobility and service characteristics so as to minimize the network traffic generated due to multicast packet delivery and multicast tree maintenance. We use $\mathrm{RBMoM}$ as a baseline case against which our URRMoM scheme is compared for performance analysis in this paper.

\section{PROTOCOL DESCRIPTION}

Our URRMoM scheme has the advantage of simplicity, scalability and efficiency. URRMoM leverages a regional mobile multicast agent (MMA) responsible for tunneling multicast packets to the FA under which the MH currently resides as long as the FA is within the regional MMA's service area. Therefore, each MMA will be a member of the multicast tree since packets multicast by the source will first be sent to MMAs in the multicast tree before they are tunneled to MHs. Each MH has only one MMA at a time with its MMA being changed from time to time as it roams in the network. Our approach thus is similar to BT except that a $\mathrm{MH}$ receives multicast data by its MMA which changes dynamically instead of from the HA which is static.

The regional service size of a regional MMA is expressed in terms of the number of subnets covered by the regional MMA. Each MH keeps a counter to record the number of subnets the MH has crossed within the service area of its MMA. When the MH crosses the boundary of a subnet, the MH will first check if the new FA of the subnet is already in the multicast group (if the FA is itself a MMA of other MHs). If the current FA is already a MMA for other MHs and thus is already a member of the multicast tree, the MMA of the MH will be updated to the current FA even though the MH is still within the service area of the last MMA. The counter in the $\mathrm{MH}$ will be reset to 0 after a MMA reset. This scenario is illustrated in Figure 1-A.

If the current FA is not in the multicast tree yet (that is, the current FA is not a MMA for other MHs), the counter in the $\mathrm{MH}$ will be incremented by one in response to the subnet crossing event. Multicast packets would be tunneled from the MMA to the current FA before they are finally forwarded to the $\mathrm{MH}$. When the $\mathrm{MH}$ moves across the regional service area such that the counter reaches the regional area size (R) and if the new FA is not an MMA itself., the new FA will subscribe to the multicast tree and become a new MMA for the MH. This scenario is illustrated in Figure 1-B. When all MHs under a MMA leave, the MMA will unsubscribe from the multicast tree. 


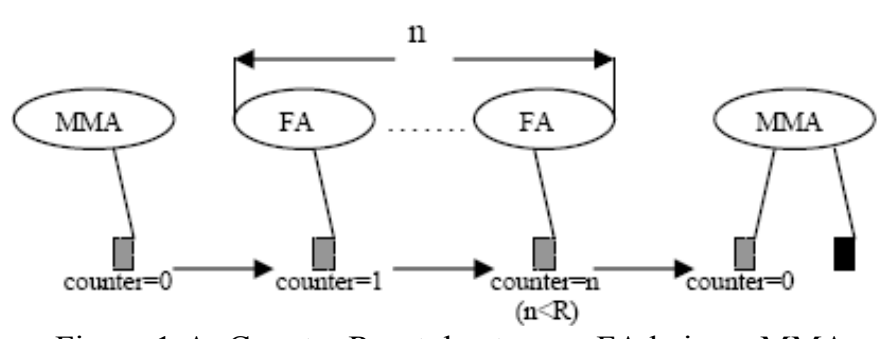

Figure 1-A: Counter Reset due to new FA being a MMA.

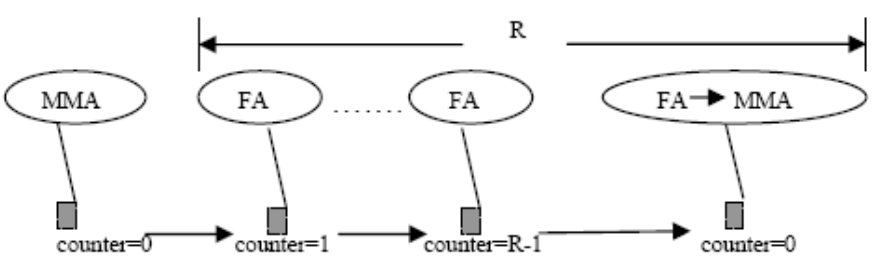

Figure 1-B: Counter Reset due to Service Area Handoff.

Under the regional registration based mobile multicast scheme, there are two types of moves:

-Intra-Regional Move: This occurs when a $\mathrm{MH}$ performs a location handoff within a multicast service area of a regional MMA. The MH's regional MMA is changed only if the new FA it enters into is itself a MMA for other MHs. In this case, the MH's MMA is updated to the current FA. Otherwise, the MH's MMA remains the same.

-Inter-Regional Move: This occurs when a MH moves across a service area (that is, the counter reaches $\mathrm{R}$ ), thus incurring a multicast service handoff. The MH's MMA will be changed. If the new FA is itself a MMA, then the MH's MMA is simply updated to the current FA. Otherwise, the current FA becomes the MH's new MMA and a multicast tree subscription event is triggered to add the new MMA to the multicast tree.

We conceive that there exists an optimal service area size that will minimize the network traffic generated due to mobile multicast services. It depends not only on the mobility and population of the MHs, but also on the size and topology of the network. Below we develop a performance model to describe the behavior of a MH operating under URRMoM..

\section{MODEL}

We consider a multicast group with a single source. The source is a fixed host (e.g., a server application). The multicast group membership does not change dynamically but mobile members may roam dynamically from one subnet to another in the mobile IP environments. Suppose that the number of MHs belonging to the multicast group is $M$. Also suppose that the network is a two-dimensional $n$ by $n$ Mesh network where each node has exactly four neighbors. A node corresponds to a subnet with a FA. A $\mathrm{MH}$ can move in four directions randomly with equal probability as shown in Figure 2.

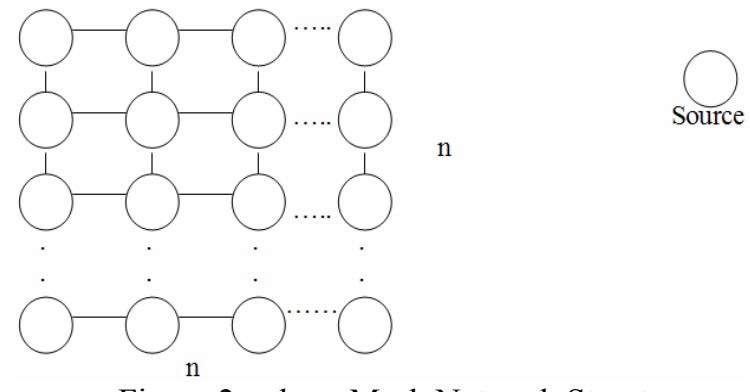

Figure 2: $n$ by $n$ Mesh Network Structure.

For simplicity, assume that a MH's residence time in FA is exponentially distributed with parameters $\mu$. It can be shown that the arrival rate of a single $\mathrm{MH}$ to any FA in this $n \times n$ homogenous network, denoted by $\lambda$, is given by [5]:

$$
\lambda=\frac{\mu}{n^{2}-1}
$$

We model the arrival-departure process of $M$ members to a $\mathrm{FA}$ as an $\mathrm{M} / \mathrm{M} / \infty / \mathrm{M}$ as shown in Figure 3 . When at least one member is in a FA, the FA is a MMA. We can obtain the probability $\left(\mathrm{P}_{0}\right)$ of a FA not covering any mobile member in the multicast group in its subnet. When a FA, acting as a MMA, does not contain any group member, it will unsubscribe from the multicast tree.

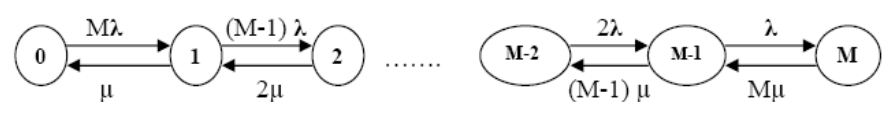

Figure 3: Arrival-Departure Process of $M$ Members with respect to a FA.

By solving a set of linear equations for $\mathrm{P}_{\mathrm{i}}$ from Figure 3, with the condition that $\sum_{i=0}^{M} P_{i}=1$, we obtain:

$$
P_{0}=\frac{1}{(1+\lambda / \mu)^{M}}
$$

Combining (1) and (2), we get:

$$
P_{0}=\left(1-1 / n^{2}\right)^{M}
$$

The average number of members in the multicast group being resided under one FA can be calculated by:

$$
\bar{n}=\sum_{i=0}^{M} i \cdot P_{i}
$$

Under our URRMoM scheme, every FA is capable of acting as a mobile multicast agent (MMA), and every $\mathrm{MH}$ keeps a counter to record how many FAs which are not a MMA it has crossed. When a MH crosses a subnet, if the new FA it just enters is already a MMA, the $\mathrm{MH}$ will consider the new FA as its MMA and reset the counter. Otherwise, it will 
increment its counter. If the counter value is equal to $\mathrm{R}$ representing the service area size of a MMA, the $\mathrm{MH}$ will subscribe the FA it just enters as a new MMA to the multicast tree and reset the counter. In our scheme, a MMA on average will cover $\mathrm{R}$ subnets, so the average number of multicast members a MMA covers is $R \cdot \bar{n}$. Thus, on average there are roughly $\frac{M}{R \bar{n}}$ MMAs in the system. Consequently, the probability that a FA that a $\mathrm{MH}$ just enters is a MMA, denoted by $\mathrm{P}_{\mathrm{MMA}}$, can be calculated by:

$$
P_{\text {MMA }}=\frac{M}{R \cdot \bar{n} \cdot n^{2}}
$$

We aim to find the optimal service size expressed in terms of the number of subnets covered by a MMA, or the optimal service size $\mathrm{R}$, such that the network traffic cost per time unit is minimized. This optimal service area size is per-MH based depending on the MH's service and mobility characteristics. This network traffic cost considered includes the cost of multicast group management and the multicasting packet delivery cost through tunneling from the MMA to the $\mathrm{MH}$. The optimal service size $\mathrm{R}$ depends on the tradeoff between the multicast group management costs vs. the tunneling cost.

We develop a stochastic Petri net (SPN) model as shown in Figure 4 to describe the behavior of a $\mathrm{MH}$ as it roams across the network. Table 1 lists places and transitions defined in the SPN model, along with their physical meanings. We determine the optimal multicast service size by evaluating the SPN parameterized by a set of parameter values characterizing the MH's service and mobility behaviors as well as the operational and workload conditions of the system.

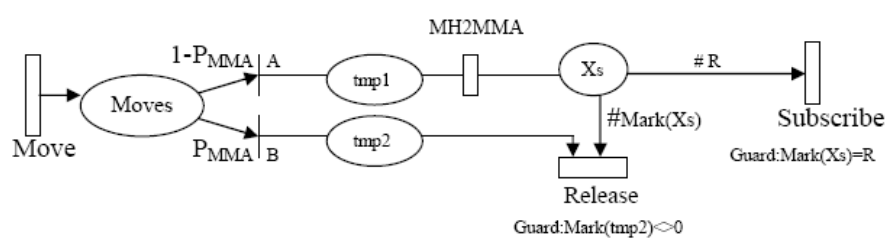

Figure 4: Performance Model for Describing a MH's Behavior.

\begin{tabular}{|l|l|}
\hline \multicolumn{1}{|c|}{ Symbol } & \multicolumn{1}{c|}{ Meaning } \\
\hline Move & $\begin{array}{l}\text { a timed transition for the MH to move across } \\
\text { a subnet areas }\end{array}$ \\
\hline Moves & $\begin{array}{l}\text { Mark(moves)=1 means that the MH just } \\
\text { moves across a subnet }\end{array}$ \\
\hline MH2MMA & $\begin{array}{l}\text { a timed transition for the MH to inform the } \\
\text { current MMA of the CoA change }\end{array}$ \\
\hline $\mathrm{P}_{\text {MMA }}$ & $\begin{array}{l}\text { probability that the FA that the MH just } \\
\text { enters is already a MMA in the multicast } \\
\text { tree }\end{array}$ \\
\hline Xs & $\begin{array}{l}\text { Mark(Xs) holds the number of subnets } \\
\text { crossed by the MH in a multicast service } \\
\text { area }\end{array}$ \\
\hline Subscribe & $\begin{array}{l}\text { a timed transition to inform the multicast } \\
\text { source that the current FA will be added into } \\
\text { the multicast tree }\end{array}$ \\
\hline R & $\begin{array}{l}\text { service area covered by a MMA (number of } \\
\text { subnets covered by a MMA) }\end{array}$ \\
\hline
\end{tabular}

\begin{tabular}{|l|l|}
\hline Release & $\begin{array}{l}\text { a timed transition for the MH to claim the } \\
\text { current FA as its MMA }\end{array}$ \\
\hline Guard:Mark(tmp2) $\neq 0$ & $\begin{array}{l}\text { a guard for transition Release that is enabled } \\
\text { if the MH moves to a subnet whose FA is } \\
\text { already a MMA }\end{array}$ \\
\hline Guard:Mark(Xs)=R & $\begin{array}{l}\text { a guard for transition Subscribe that is } \\
\text { enabled if the number of tokens in place Xs } \\
\text { is equal to R }\end{array}$ \\
\hline
\end{tabular}

Table 1: Meaning of Places and Transitions in the SPN Model.

A token in the SPN model represents a subnet crossing event by the $\mathrm{MH}$. The function Mark(D) returns the number of tokens in place D. Mark(Xs) represents the number of subnets already crossed by the $\mathrm{MH}$ since the previous MMA reset. Our SPN model allows the event time associated with a transition to be exponentially or generally distributed so the underlying state model can be a very general semi-Markov chain [7]. Below we describe how we construct the SPN model to describe the behavior of a $\mathrm{MH}$ operating under our scheme:

- When a MH moves across a subnet area, it incurs a location handoff. This is modeled by placing a token in place "Moves." The mobility rate at which location handoffs occur is $\mu$ which is the transition rate assigned to transition "Move".

- If the MH moves to a new FA which is not a MMA, the probability of which is $1-\mathrm{P}_{\mathrm{MMA}}$, then transition $\mathrm{A}$ will be fired immediately to move the token in place "Moves" to place tmp1. The MH will communicate with its current MMA to update its current CoA. This is modeled by enabling and firing transition MH2MMA. After MH2MMA is fired, a token in place tmp1 flows to place $\mathrm{Xs}$, representing that a location handoff has been completed. When the $\mathrm{MH}$ has made $\mathrm{R}$ moves, i.e., the number of tokens in place $\mathrm{Xs}$ reaches $\mathrm{R}$, it means that the $\mathrm{MH}$ has moved out of the current service area covered by the current MMA, so the FA which the MH just moved into will become a MMA (if it is not already a MMA itself) and a new subscription request will be sent to the source to add the new MMA to the multicast tree. This is modeled by firing transition "Subscribe," after which all $\mathrm{R}$ tokens are consumed and place Xs contains zero token.

- If the MH moves to a FA which is already a MMA, the probability of which is $\mathrm{P}_{\mathrm{MMA}}$, then transition $\mathrm{B}$ will be fired immediately to move the token in place "Moves" to place tmp2. Subsequently the timed transition "Release" will be fired to consume all tokens in both tmp2 and Xs. This models the fact that the counter kept by the $\mathrm{MH}$ is reset to 0 and the current FA becomes the new MMA for the $\mathrm{MH}$.

\begin{tabular}{|l|l|}
\hline \multicolumn{1}{|c|}{ Symbol } & \multicolumn{1}{c|}{ Meaning } \\
\hline$\lambda$ & arrival rate of a MH to any FA in the $\mathrm{n}$ by $\mathrm{n}$ network \\
\hline$\mu$ & MH's departure rate in a FA \\
\hline $\mathrm{M}$ & number of MHs in the multicast group \\
\hline $\mathrm{P}_{\mathrm{i}}$ & probability of state i in Figure 3. \\
\hline$\lambda_{\mathrm{m}}$ & $\begin{array}{l}\text { number of join or leave operations to the multicast } \\
\text { tree per unit time }\end{array}$ \\
\hline$\lambda_{\mathrm{p}}$ & number of multicast packets delivered per unit time \\
\hline$\beta$ & average number of hops to reach the source for \\
\hline
\end{tabular}




\begin{tabular}{|l|l|}
\hline & multicast tree subscription/un-subscription \\
\hline $\mathrm{R}$ & $\begin{array}{l}\text { number of subnets (or FAs) in one service area } \\
\text { covered by a MMA }\end{array}$ \\
\hline $\bar{n}$ & $\begin{array}{l}\text { average number of MHs in the multicast group in } \\
\text { one FA }\end{array}$ \\
\hline $\mathrm{r}_{\text {sub }}$ & $\begin{array}{l}\text { transition rate of "Subscribe" } \\
\text { probability of state i in the underlying Markov or } \\
\text { semi-Markov model of the SPN model in Figure 4 }\end{array}$ \\
\hline$\tau$ & 1-hop communication delay in wired networks \\
\hline \multicolumn{1}{|c}{ Table 2: Parameters. } \\
\hline
\end{tabular}

Our performance metric is the total cost incurred per time unit due to multicast tree maintenance and multicast packet delivery. The total overhead cost $\mathrm{C}_{\text {Total }}$ is defined as:

$$
C_{\text {Total }}=C_{\text {Maintenance }}+C_{\text {Service }}
$$

where $\mathrm{C}_{\text {Maintenance }}$ is the cost incurred per unit time due to control packets for tree management and $\mathrm{C}_{\text {Service }}$ is the cost per unit time for delivering multicast packets from the multicast source to MHs in the multicast group.

The optimal service size covered by a MMA can be determined by considering the tradeoff between $\mathrm{C}_{\text {Maintenance }}$ vs. $\mathrm{C}_{\text {Service. }}$. We expect that $\mathrm{C}_{\text {Maintenance }}$ will increase and $\mathrm{C}_{\text {Service }}$ will decrease as $\mathrm{R}$ decreases. On the other hand, as $\mathrm{R}$ increases, $\mathrm{C}_{\text {Maintenance }}$ will decrease but $\mathrm{C}_{\text {Service }}$ will increase. Our goal is find the optimal service area when given a set of parameter values characterizing the operating and workload conditions.

A multicast tree operation occurs when a new MMA is subscribed to the tree, or when an existing MMA is unsubscribed from the tree. We subscribe a new MFA to the tree with the rate of $r_{\text {sub }} \cdot M$ where $r_{\text {sub }}$ is the rate at which a member subscribes a new MMA to the multicast tree after it has crossed $\mathrm{R}$ subnets; this subscription rate corresponds to the transition rate of transition "Subscribe" in the SPN model.

We unsubscribe an existing MMA from the multicast tree when the MMA no longer covers any multicast member in its region, the rate of which is given by $P_{1} \cdot \mu$ where $\mathrm{P}_{1}$ is the probability that the MMA is in state 1 in Figure 3 and $\mu$ is the rate at which the last MH leaves the MMA. Let $\beta$ denote the average number of hops separating a MMA and the multicast source. Let $\tau$ be the average per-hop communication cost. A tree subscription/un-subscription operation thus costs $\beta \tau$. We then compute $\mathrm{C}_{\text {Maintenance }}$ by the cost per operation multiplied with the rate at which the operation is generated, i.e.,

$$
C_{\text {Ma intenance }}=\lambda_{m} \beta \tau
$$

with

$$
\lambda_{m}=P_{1} \cdot \mu \cdot\left(\frac{M}{R n}\right)+r_{\text {sub }} \cdot M
$$

In Equation 8, $\frac{M}{R \bar{n}}$ is the average number of MMAs in the system, $P_{1} \cdot \mu \cdot\left(\frac{M}{R \bar{n}}\right)$ is the number of multicast tree unsubscribe operations per unit time, and $r_{\text {sub }} \cdot M$ is the number of multicast tree subscribe operations per unit time.

The cost for multicast packet delivery per unit time, $\mathrm{C}_{\text {Service, }}$, is given by the cost per packet delivery multiplied with the rate at which packets are generated, i.e.,

$$
C_{\text {Service }}=\lambda_{p}\left\{\beta \cdot \frac{M}{R \cdot \bar{n}}+M \sum_{i=0}^{R}\left(q_{i} \cdot i\right)\right\} \cdot \tau
$$

In Equation 9, $\beta \cdot \frac{M}{R \bar{n}}$ is the number of hops for multicast packet delivery from the multicast source to MMAs, and $M \sum_{i=0}^{R}\left(q_{i} \cdot i\right)$ is the number of hops through which packets are tunneled from various MMAs to $M$ MHs. The steady-state probability of state $\mathrm{i}, q_{\mathrm{i}}, 1 \leq \mathrm{i} \leq \mathrm{R}$, needed in Equation 9 can be solved easily from our SPN model utilizing solution techniques such as SOR or Gauss Seidel [7].

\section{NUMERIC DATA AND ANALYSIS}

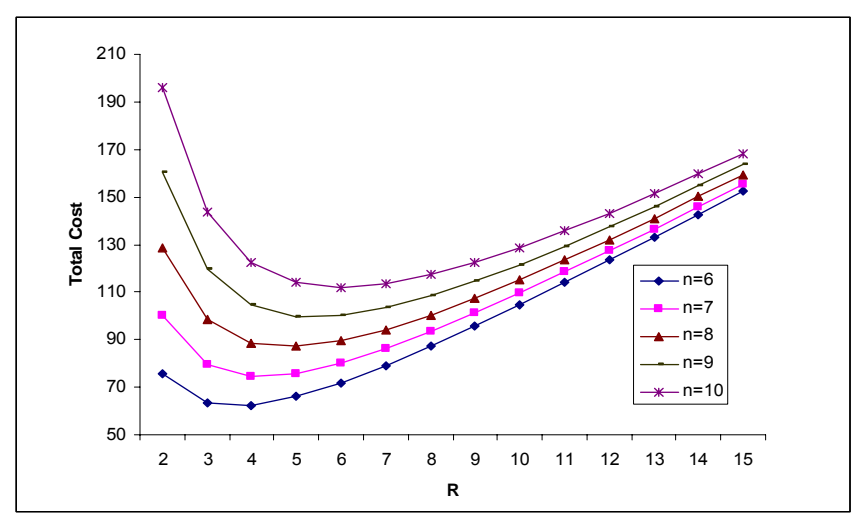

Figure 5: Cost vs. Regional Area Size (R) with varying $n$.

As a case study, we set $M=100, \tau=0.025 \mathrm{~s}$ and $\lambda_{\mathrm{p}}=10$ while we vary values of other parameters to study their effects on system performance. Figure 5 shows the total traffic generated as a function of the service area size $\mathrm{R}$ expressed in terms of the number of subnets for the case in which $\beta=15, \mu=0.00167$ (MH's residence time in one FA is 10 minutes) and $\mathrm{M}=100$. We see that there exists an optimal service area size under which the network traffic generated is minimized. Also, as the mesh network becomes larger (as $n$ increases), the optimal service area size becomes larger and larger.

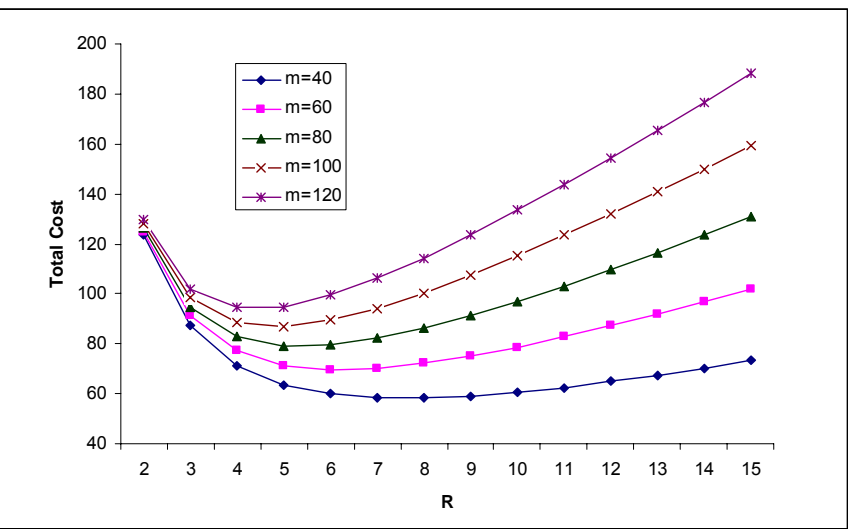

Figure 6: Cost vs. R with Varying Number of MHs.

Figure 6 shows the network traffic generated vs. R as $M$ (the number of MHs in the multicast group) varies in an 8 by 8 mesh network. We see that as $\mathrm{M}$ increases the optimal $\mathrm{R}$ 
decreases. The reason is that as $\mathrm{M}$ increases, the multicast packet delivery cost dominates the multicast tree maintenance cost, so the system prefers to have a small service area to reduce the multicast packet delivery cost by routing packets through shorter MMA-MH routes.

Figure 7 shows that when $\beta$ increases, the optimal range $R$ increases (for the case when $\mathrm{M}$ is fixed at 100). The reason is that as the distance separating the source and MMA increases, the maintenance cost increases, so the system prefers to have a larger service area to reduce the rate of tree subscription/unsubscription operations.

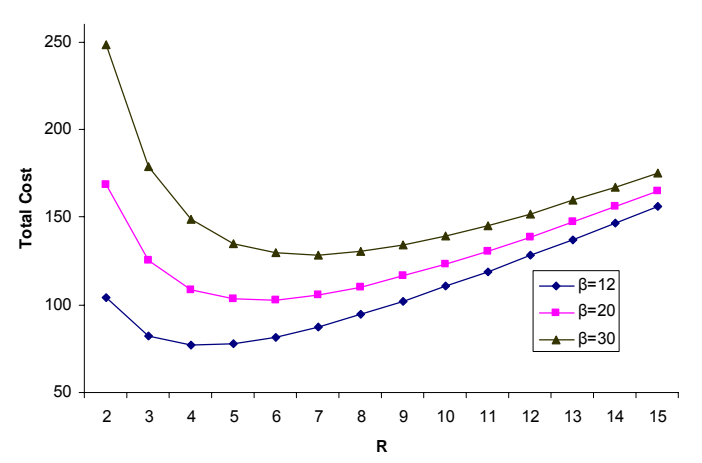

Figure 7: Effect of the Distance between Source and MMA.

We perform a comparative analysis of URRMoM with RS and RBMoM to demonstrate the benefit of URRMoM. The RS scheme is a special case by setting our regional area size R to one since on every subnet boundary crossing, the $\mathrm{MH}$ will subscribe to the multicast source directly. The RBMoM scheme is based on range, so in spirit the service range size is similar to the service area size in our scheme except that when a FA becomes a MMA it needs to inform all FAs located within the service range. Also, a MH under RBMoM will not change its MMA until it crosses the service range of the MMA. When a MH crosses the service range of the current MMA, it needs to check with the new FA of the closest MMA to register to it. The multicast maintenance cost per unit time is therefore given by:

$$
C_{\text {maintenance }}^{\text {RBMo }}=P_{1} \cdot \mu \cdot\left(\frac{M}{R n}\right) \beta \tau+r_{\text {sub }} \cdot M \cdot\left[\beta+\left(\sum_{j=1}^{R} j\right)\right] \cdot \tau
$$

Here the first term is the cost per unit time for multicast tree un-subscription. The second term is the cost per unit time for multicast tree subscription. When a multicast tree subscription event occurs, the cost includes a subscription packet sent from the FA to the multicast source (which costs $\beta \tau$ ) and a packet sent from the new MMA to inform its presence to other FAs in its area. The service cost per unit time under RBMoM is the same as that given for URRMoM in Equation 9.

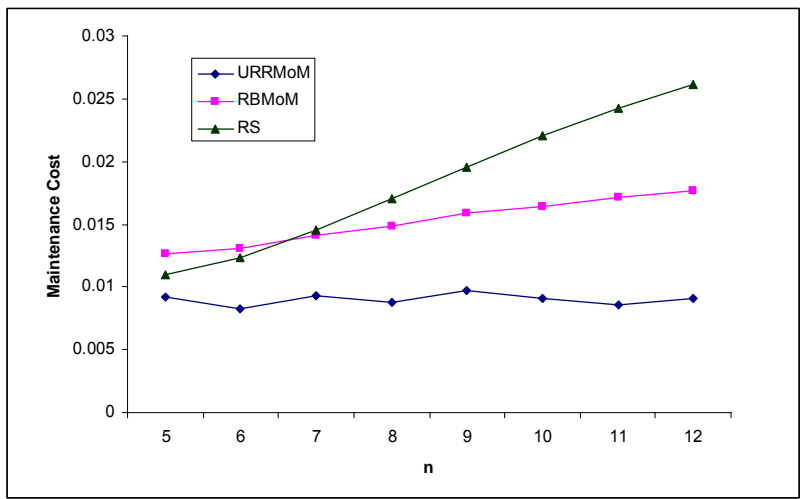

Figure 8: Comparison of URRMoM vs. RS and RBMoM.

Figure 8 compares the network traffic generated due to maintenance vs. the network size $n$ for URRMoM vs. RS and RBMoM at optimizing $\mathrm{R}$ values under the same set of parameter values. We see that URRMoM always produces the least amount of network traffic compared with RS and RBMoM. The reason is that RS is just a special case in which $\mathrm{R}=1$, while URRMoM incurs the extra overhead of the new MMA informing FAs in its area. Our URRMoM scheme is user-oriented without requiring any MH to inquiry a FA of the nearest MMA. Rather, each MH will simply keep a counter to help it decide if it should make the current FA as its MMA when the MMA service area is crossed, provided that the current FA is itself not a MMA. This mechanism greatly reduces the cost for mobile multicast service management.

\section{Simulation}

We have conducted an extensive simulation study using a discrete event simulation language called SMPL (Simulation Model Programming Language) [8] to validate the analytical results reported in Section V. To ensure statistical significance of simulation results, a batch mean analysis (BMA) technique has been adopted by which the simulation period is divided into batch runs with each batch consisting of 2,000 "cost rate" observations for computing an average value. A minimum of 10 batches was run to compute a grand mean of the cost rate value. Additional batches were added if necessary until the mean cost rate value is within $95 \%$ confidence level and $10 \%$ accuracy from the true mean.

The simulation results show very good correlations with analytical results. For example, Figures 9 and 10 show the simulation results for the total cost vs. R with varying $n$ and varying $m$, respectively, corresponding to Figures 5 and 6 for analytical results in Section V. We see that Figures 9 and 10 are virtually identical to Figures 5 and 6 in shape despite the fact that simulation results are obtained based on the cumulative cost over the simulation period in response to multicast tree subscribe/unsubscribe and multicast packet delivery service events divided by the simulation period, while analytical results are obtained based on the average cost rate as calculated by Equations (6), (7) and (9) following the parameterization process. We conclude that the analytical results are valid and there exists an optimal regional area size under which URRMoM is optimized. 


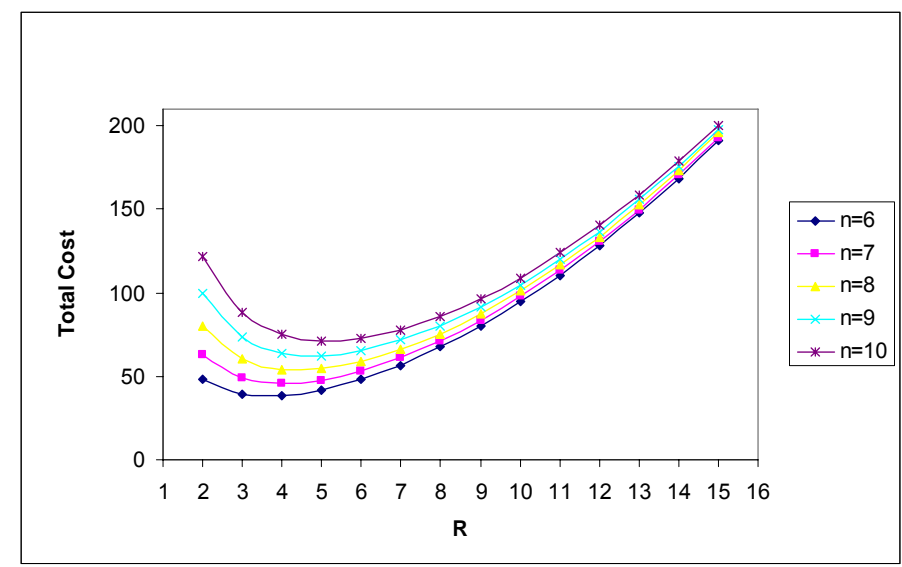

Figure 9: Simulation Results - Cost vs. R with varying $n$.

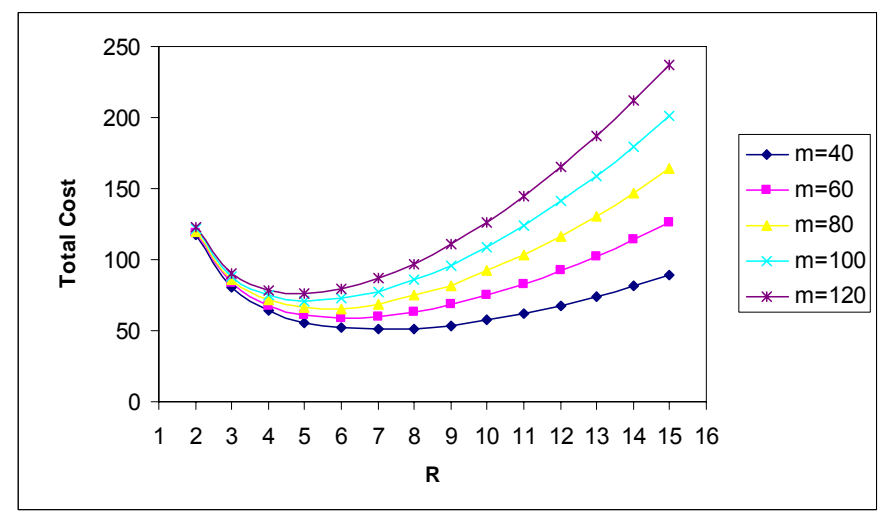

Figure 10: Simulation Results - Cost vs. $\mathrm{R}$ with varying Number of MHs.

We have also simulated RS (a special case of URRMoM in which $\mathrm{R}=1$ ) and $\mathrm{RBMoM}$ and conducted a simulation study to compare URRMoM with RS and RBMoM. We utilize the BMA technique to obtain the mean multicast tree maintenance cost rate with statistical significance for these three schemes. Figure 11 shows the simulation results of multicast tree maintenance cost vs. R, corresponding to Figure 8 for analytical results. We see that Figure 11 again correlates very well with Figure 8, confirming the analytical results that URRMoM always produces the least amount of network traffic compared with RS and RBMoM.

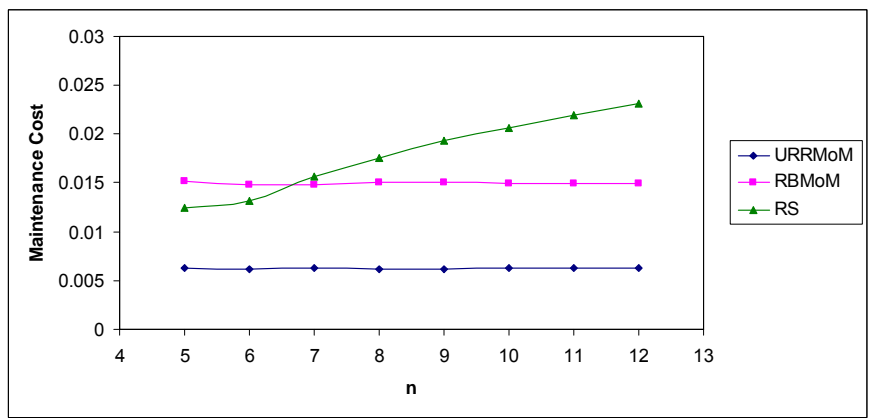

Figure 11: Simulation Results - Comparison of URRMoM vs. RS and RBMoM.

\section{CONCLUSION AND FUTURE WORK}

In this paper, we have proposed and analyzed a useroriented regional registration based mobile multicast (URRMoM) approach for supporting mobile multicast services in Mobile IP environments. Our approach combines distinct performance advantages of remote subscription and bi-directional tunneling. URRMoM also has simpler system requirements and less computation complexity than RBMoM. We developed a mathematical model to analytically determine the optimal service area size under which the overall network traffic generated due to multicast tree maintenance and multicast packet delivery can be minimized. We also analyzed the effect of key parameters on the optimal regional area size and provided reasons for the sensitivity analysis. A comparative analysis was performed analytically and by simulation to demonstrate the benefit of URRMoM compared with existing schemes including RS and RBMoM for mobile multicast management. In the future, we plan to perform empirical validation of URRMoM in an experimental testbed.

\section{Acknowledgement}

Thanks to Heng Zhang, a MS graduate at Virginia Tech, for writing code to evaluate the SPN model for numerical analysis and Jennifer Wang for writing simulation code in smpl to validate analytical results.

\section{REFERENCES}

[1] I.R. Chen, W. He, and B. Gu, "DMAP: Integrated mobility and service management in Mobile IPv6 systems," Wireless Personal Communications, 2007.

[2] L.C. Richard and W. Kai-Min, "Scalable multicast protocol in IP-based mobile networks," ACM/Springer Wireless Networks, vol. 8, pp. 27-36, 2002.

[3] C. Perkins, IP Mobility Support, IETF RFC 3344, 2007.

[4] H. Omar, T. Saadawi and M. Lee, "Multicast support for Mobile IP with the hierarchical local registration approach," 3rd ACM International Workshop on Wireless Mobile Multimedia, Boston, Massachusetts, 2000.

[5] H. Yungoo and K. Cheeha, "mMOM: efficient mobile multicast support based on the mobility of mobile hosts," ACM/Springer Wireless Networks., vol. 12, pp. 171-178, 2006.

[6] Y. Wang and W. Chen, "Supporting IP multicast for mobile hosts," ACM/Springer Mobile Networks and Applications, vol. 6, pp. 57-66, 2001.

[7] K. Trivedi, G. Ciardo, and J. Muppala, SPNP Version 6 User Manual, Duke University, Durham, NC, 1999.

[8] M.H. MacDougall, Simulating Computer Systems. MIT Press, 1987. 